Revista de Psicologia de la PUCP. Vol. XV, 1, 1997.

\title{
EL DESARROLLO DE LAS HABILIDADES COGNITIVAS SEGÚN LOS AVANCES EN LAS TEORÍAS PSICOLÓGICAS
}

\author{
Sheyla Blumen ${ }^{1}$ \\ Pontificia Universidad Católica del Perú
}

Se presentan los modelos más representativos del desarrollo cognitivo según los avances en las teorias psicológicas. Luego se realiza un breve análisis de los modelos en función a seis factores relacionados con diferentes áreas de la psicología y las ciencias sociales (importancia de cada etapa, procesos, conocimiento, diferencias individuales, contexto y limitaciones en el desarrollo cognitivo). Finalmente se presenta una propuesta de integración de los modelos actuales del desarrollo cognitivo desarrollado por Sincoff y Sternberg (1989).

Palabras claves: habilidades cognitivas, cognición, desarrollo cognitivo, teorías cognitivas.

The development of cognitive abilities following the new outcomes of psychological theories. The most representative models of cognitive development following the new outcomes of psychological theories are presented. Then a brief analysis of the models in terms of six factors related to different areas in psychology and social sciences (importance of each stage, processes, knowledge, individual differences, context and limits in the cognitive development) is developed. Finally, an integration of the model developed by Sincoff and Sternberg (1989) is presented.

Key words: cognitive abilities, cognition, cognitive development, cognitive theories.

1. Docente de la Sección de Psicología del Departamento de Humanidades de la Pontificia Universidad Católica del Perú. Apartado 1761, Lima 100 - Perú; Internet: sblumen@pucp.edu.pe. Es delegada del World Council of Gifted and Talented Children y Representante del European Council of High Abilities. Expositora en diversos eventos académicos a nivel nacional e internacional, es candidata al doctorado en Psicología en la Universidad Católica de Nijmegen (Holanda). Sus intereses se relacionan con la psicología y educación de los niños con altas capacidades y talentosos. 

Los niños, en su búsqueda contínua de respuestas respecto a los eventos o situaciones que ocurren en el mundo que los rodea, no se satisfacen tan sólo con observar un evento, sino que desean saber cómo o por qué ocurren las cosas. Los psicólogos que estudian el desarrollo cognitivo de los niños se encuentran con los mismos problemas, mientras que para algunos investigadores es suficiente el identificar los niveles por los cuales los niños atraviesan durante su desarrollo intelectual, para otros, especialmente para los actuales teóricos e investigadores, la pregunta más importante está relacionada con el cómo o por qué los niños pasan de un nivel o estadio a otro. Estos psicólogos buscan identificar los procesos o mecanismos a través de los cuales se presenta el desarrollo cognitivo en el niño.

Si bien el interés en la investigación sobre los mecanismos de desarrollo del pensamiento presenta sus inicios en el importante trabajo de Jean Piaget (1896-1980), la mayoría de la investigación conducida por Piaget y sus sucesores estuvo diseñada para proveer descripciones detalladas sobre los estados diferenciados del pensamiento de los niños en diferentes edades o niveles de desarrollo y, con los avances metodológicos y conceptuales de la actualidad, el interés se ha centrado en proporcionar información detallada sobre los mecanismos responsables del desarrollo cognitivo.

Las teorías sobre el desarrollo cognitivo han progresado significativamente en los últimos años, con teóricos como Case, Fischer, Klahr, Siegler, Sternberg, Keil y Carey (Case, 1985) entre otros, quienes han introducido modelos parciales acerca del desarrollo cognitivo. Debido a la proliferación de modelos, es importante examinar algunos de éstos, y su influencia en nuestra comprensión sobre el desarrollo de las habilidades de pensamiento. Para ello desarrollaremos primero una breve descripción de los nuevos modelos propuestos en función a seis factores relacionados con diferentes áreas de la psicología y las ciencias sociales (importancia de 
cada etapa, procesos, conocimiento, diferencias individuales, contexto y limitaciones en el desarrollo cognitivo) y segundo se presentará una propuesta de integración de los modelos desarrollada por Sincoff y Sternberg (1989).

En un intento de explicar la naturaleza de los mecanismos de desarrollo cognitivo los actuales teóricos del desarrollo cognitivo han intentado presentar alternativas a las propuestas de Werner, Vygotsky y Piaget. Estas se encuentran resumidas en el Cuadro 1.

\section{Cuadro 1}

Resumen de los modelos de desarrollo

\begin{tabular}{|c|l|}
\hline NVESTIGADOR & \multicolumn{1}{|c|}{ PROPUESTA } \\
\hline $\begin{array}{c}\text { Robbie Case } \\
(1985)\end{array}$ & $\begin{array}{l}\text { Presenta un modo neopiagetano de desarrollo influenciado por las áreas } \\
\text { de procesamiento de la información y la simulación computarizada. }\end{array}$ \\
\hline $\begin{array}{c}\text { Kurt Fischer } \\
(1980)\end{array}$ & $\begin{array}{l}\text { Propone una teoría de etapas de destrezas de aprendizaje, en la que el } \\
\text { pensamiento del niño se desarrolla a través de la activación de cinco } \\
\text { normas de transformación. }\end{array}$ \\
\hline $\begin{array}{c}\text { David Klahr } \\
(1984)\end{array}$ & $\begin{array}{l}\text { Utiliza sistemas de producción de auto-modificación y otros conceptos } \\
\text { de procesamiento de información para explicar el desarrollo cognitivo, } \\
\text { especialmente la adquisición de constructos piagetanos como la con- } \\
\text { servación. }\end{array}$ \\
\hline $\begin{array}{c}\text { Robert Siegler } \\
(1986)\end{array}$ & $\begin{array}{l}\text { Propone un modelo de procesamiento de la información para explicar } \\
\text { el desarrollo de las destrezas piagetanas y otras destrezas intelectuales } \\
\text { superiores. }\end{array}$ \\
\hline $\begin{array}{c}\text { Robert Sternberg } \\
(1985)\end{array}$ & $\begin{array}{l}\text { Influenciado por los campos de procesamiento de la información y las } \\
\text { habilidades humanas, propone una teoría triárquica de la inteligencia } \\
\text { y su desarrollo, entapizando las relaciones entre los componentes in- } \\
\text { ternos del niño referidos al procesamiento de la información, su ex- } \\
\text { periencia y su ambiente. }\end{array}$ \\
\hline $\begin{array}{c}\text { Fusan Carey } \\
(1985)\end{array}$ & $\begin{array}{l}\text { Influenciado por los trabajos de Chomsky y Fodor, señala que el } \\
\text { desarrollo proviene de procesos cognitivos generales, que reestructuran } \\
\text { el conocimiento específico. }\end{array}$ \\
\hline $\begin{array}{l}\text { Enfatiza la importancia crucial de las estructuras del conocimiento de } \\
\text { dinainio específico y representacionales. Señala que el desarrollo } \\
\text { cognitivo consiste en la emergencia de nuevas estructuras de conoci- } \\
\text { miento a partir de la reorganización y expansión de otras más antiguas. }\end{array}$ \\
\hline
\end{tabular}


Estos teóricos contemporáneos preocupados respecto al desarrollo cognitivo han desarrollado modelos para explicar sus propuestas. A continuación se presentarán los modelos de los siete teóricos del desarrollo cognitivo, en función a sus propuestas respecto a seis factores importantes del desarrollo cognitivo:

- Primero, las etapas o estadios han ayudado a representar los principios organizacionales de las teorías del desarrollo cognitivo y han presentado diferentes funciones heurísticas. Se analizará si estos nuevos modelos ofrecen o no algunos principios guía para caracterizar el pensamiento de los niños.

- Segundo, los avances en la psicología del procesamiento de la información han dirigido su atención a la importancia de explicar los procesos que están a la base del cambio cognitivo. Se analizará el tipo de procesos que proponen las nuevas teorías para explicar el desarrollo cognitivo.

- Tercero, las estructuras de conocimiento y las representaciones mentales han sido tradicionalmente el centro de atención en las teorías de desarrollo cognitivo así como en la psicología de procesamiento de la información. Se analizará el rol del conocimiento en las nuevas teorías del desarrollo.

- Cuarto, los psicometristas y psicólogos de la información han provisto gran cantidad de información sobre las diferencias individuales en el desempeño cognitivo. Se analizará si las nuevas teorías explican las diferencias individuales en la adquisición y logro de las habilidades cognitivas.

- Quinto, el trabajo en diferentes áreas ha puesto en relieve la importancia de estudiar el desarrollo dentro de un marco contextual. Vygotsky resaltó la influencia del entorno social del niño y los antropólogos han estudiado siempre la conducta dentro de su contexto cultural. Se analizará el rol del contexto en las nuevas teorías del desarrollo.

- Finalmente, las teorías del desarrollo cognitivo tradicionales, la psicología del procesamiento de la información y la biología evo- 
lutiva han señalado limitaciones en el desarrollo cognitivo. Se analizarán las limitaciones en el desarrollo cognitivo propuestas por las nuevas teorías.

\section{El principio organizador del desarrollo}

En el proceso de estudio de la estructura de pensamiento del niño y en los procesos que están a la base de los cambios estructurales, los teóricos del desarrollo cognitivo señalan también, de manera explícita o implícita la organización de los cambios que ocurren mientras el niño se desarrolla. Si bien las etapas o estadios representan el principio de organización del desarrollo cognitivo tradicional, en la actualidad se han propuesto otros principios guía.

En términos generales, las teorías de los estadios suponen cuatro características de cambio estructural (Kohlberg, 1969). En primer lugar, los estadios suponen modos de pensamiento cualitativamente discretos, en diferentes niveles de edad. En segundo lugar, los modos de pensamiento cualitativamente discretos forman una secuencia invariante en el curso del desarrollo. En tercer lugar, los modos de pensamiento característicos de cualquier etapa particular están relacionados y forman juntos una estructura (un todo). Finalmente, las etapas o estadios son jerárquicos: estadios más avanzados se reintegran y finalmente suplantan o desplazan a modos de pensamiento de niveles menores. Piaget y Vygotsky fueron teóricos de estadios. Case y Fischer, cada uno adoptando una aproximación neopiagetana en el estudio del desarrollo intelectual, también ofrecen teorías de estadios de cambio cognitivo.

Case y Fischer son "piagetanos" porque asumen que el desarrollo del pensamiento en el niño se da en etapas. Pero son "neo" dado que proponen diferentes estructuras y procesos de aquellos propuestos por Piaget. Si bien los estadios propuestos por Case son similares a los de Piaget, no son idénticos.

Case (1985) propone cuatro estadios que van de la infancia hasta la adolescencia (ver Cuadro 2). 
Habilidades cognitivas

Cuadro 2

Estadios según Case

\begin{tabular}{|l|c|}
\hline $\begin{array}{c}\text { Estadios propuestos por } \\
\text { Case }(1985)\end{array}$ & $\begin{array}{c}\text { Edades } \\
\text { aproximadas }\end{array}$ \\
\hline Estadio Sensoriomotor & Nacimiento a 18 meses \\
\hline Estadio Relacional & 18 meses a 5 años \\
\hline Estadio Dimensional & 5 años a 11 años \\
\hline $\begin{array}{l}\text { Estadio Vectorial o } \\
\text { Dimensional-Abstracto }\end{array}$ & 11 años a 18 ó 19 años \\
\hline
\end{tabular}

La actividad mental de los infantes en el estadio sensoriomotor se presenta entre la acción perceptual y física. Los modos de pensamiento característicos del estadio relacional se centran en las relaciones entre o intra eventos. Los niños en el estadio dimensional se centran en las numerosas dimensiones de los objetos concretos, tales como peso o número, mientras que los adolescentes en el estadio vectorial se centran en dimensiones más abstractas o de segundo orden.

Por ejemplo, para resolver una analogía, un adolescente debe encontrar una dimensión de orden superior o vector que relacione la dimensión de menor orden en la analogía, permitiéndole comparar las dimensiones de menor orden en un plano más abstracto. Case asegura que los niños pasan a través de la misma secuencia de etapas y subetapas a través de diferentes contenidos, que lo hacen al mismo ritmo y durante el mismo rango de edad. Case formula sus etapas para intentar corregir el problema de desnivel horizontal que propuso Piaget.

Case propone también que dentro de cada etapa se da el progreso en el desarrollo a través de las cuatro subetapas en las que el niño opera simultáneamente. Diferentes tipos de unidades representacionales caracterizan diferentes etapas y la transición entre las etapas ocurre cuando los problemas cuyas representaciones originalmente requieren cuatro uni- 
dades son re-representados utilizando sólo una unidad del siguiente nivel cognitivo.

Case aplica un nivel relativamente global de análisis al estudio de las representaciones de los niños. En general, él pregunta cómo los niños representan los problemas y cómo actúan al representarlos para resolverlos. Un niño sensoriomotor, por ejemplo deberá representar el trueno como un ruido fuerte (sensorial) y puede actuar su representación a través del gateo o acercamiento hacia su madre (otro).

Es en este nivel que Case demuestra las consistencias en el razonamiento de los niños a través de una variedad de tareas. Si bien señala la utilidad de este nivel de análisis global para eliminar el décalage horizontal, corre el riesgo de encontrar semejanzas entre operaciones que no son comparables, exagerando el grado de regularidad horizontal (Siegler, 1986b).

Otra dificultad en el nivel analítico global de Case es que las operaciones únicas para cada etapa son difíciles de describir. Las operaciones características de las etapas o estadios de Case son claramente similares a las de Piaget. Sin embargo, dado el nivel general que Case emplea para examinar las representaciones de los niños, parece difícil establecer exactamente qué operaciones sensoriomotoras, relacionales, dimensionales y vectoriales se presentan sin recurrir a la teoría Piagetana. Finalmente, Case no indica claramente el por qué las re-representaciones ocurren solamente luego que las cuatro unidades representacionales han sido consolidadas (Siegler, 1986b), ni logra explicar por qué es cuatro el número que emplea.

Fischer (Fischer y Pipp, 1984) se diferencia de Piaget y Case al presentar diez niveles de habilidades cognitivas cualitativamente diferentes, jerárquicamente organizadas y muy relacionadas con la edad:

un conjunto sensoriomotor,

un mapeo sensoriomotor,

un sistema sensoriomotor,

- un sistema de sistemas sensoriomotores o conjunto representacional, un mapeo representacional, 
- un sistema representacional,

- un sistema de sistemas representacionales o conjunto abstracto,

- un mapeo abstracto,

- un sistema abstracto y

- un sistema de sistemas abstractos o principio único.

A nivel empírico se presenta la emergencia de nuevos niveles, pero a nivel teórico, el nivel de habilidad óptima del niño limita sus habilidades de procesamiento de información y lo mantiene a un determinado nivel de funcionamiento.

El desarrollo se presenta a través de la activación de una norma de transformación que lleva al niño al siguiente estadio, estableciendo un nuevo nivel de habilidad óptima. Fischer sostiene que los niveles óptimos sólo establecen el límite (techo) de las habilidades y destrezas del niño, sin embargo su desempeño se presenta por lo general por debajo de tal límite. Esta aseveración representa por lo tanto, otra diferencia de Case y Piaget, y una fortaleza para la teoría de Fischer.

Para Case y Piaget, los niveles superiores desplazan a los inferiores, sin embargo, para Fischer los niveles superiores no necesariamente desplazan a los inferiores, sino que es la habilidad óptima (nivel techo) la que varía, aunque se pueden presentar conductas por debajo de la misma.

Las principales dificultades con la Teoría de Estadios de Desarrollo de Fischer son las siguientes: (a) es una teoría que postula diez niveles cualitativamente distintos de habilidades cognitivas y (b) si bien Fischer ha estudiado otras habilidades, la manera como ilustra estas etapas es a través del análisis de las habilidades de los niños en el juego con muñecas (Fischer y Pipp, 1984).

Por ejemplo, un niño que opera al nivel representacional único puede tratar a la muńeca como un doctor, haciendo que la muńeca se ponga la bata del doctor o escriba una prescripción médica. Un niño que opera al nivel de mapeo representacional puede coordinar dos roles de conducta relacionados para crear un rol social, por ejemplo haciendo que la muñeca 
doctor y la muñeca paciente interactúen de manera estereotípica. Un niño que se desenvuelve a nivel de sistema representacional puede hacer que las dos muñecas interactúen los roles sociales de manera simultánea, por ejemplo, una muñeca actúa como doctor y esposo y la segunda como paciente y esposa.

Si bien los estadios de Fischer parecen apropiados para esta instancia de juego, puede no ser apropiado para el desempeño en otras actividades. Tal como señala Flavell (1984), es difícil comprender el significado preciso, de dominio-invariante de un sistema de sistemas representacionales equivalente a un conjunto abstracto único. Por lo tanto, es difícil aplicar los niveles de Fischer a áreas diferentes a las del juego de roles. Aparte de Case y Fisher, ninguno de los teóricos que analizaremos considera estadios en sus modelos.

El segundo principio organizacional supone una organización ramificada similar a la propuesta por Werner, que sigue el modelo de desarrollo cognitivo de Carey. Según Carey (1985b), el desarrollo progresa de la misma manera como las teorías del mundo se van especializando a partir de una o dos teorías generales. Es decir, el niño inicialmente posee tan sólo teorías mecánicas y psicológicas de menor nivel, y, mientras se desarrolla, las teorías de psicología, mecánica, materia, biología, religión, economía, historia, política, etc., se van desarrollando a partir de las dos primeras teorías (Carey, 1985b). Carey propone una ramificación pequeña, dado que creía que sólo una docena de teorías emergía en el curso del desarrollo.

El tercer principio organizacional que caracteriza el desarrollo en numerosas teorías recientes se representa a través de ciclos continuos o "loops" de retroalimentación. Este principio guía se está volviendo aparentemente muy popular en el desarrollo cognitivo desde la perspectiva del procesamiento de la información. En los ciclos o "loops" de retroinformación el progreso en la estructura o proceso $x$ conlleva al progreso en la estructura o proceso $y$, que a su vez dirige el progreso en $x$, y el ciclo continúa repetidamente. Siegler (1986a), apoya esta propuesta de cambio cognitivo. 
Siegler sostiene que el conocimiento predictivo del mundo en el niño y la codificación de rasgos o características del ambiente se da en el mismo nivel. Luego, los nifios empiezan a codificar las características o rasgos faltantes, y su mejora en la codificación les permite aprender reglas y normas sofisticadas, así como avanzar en su conocimiento del mundo.

El mayor nivel de conocimiento forma la base para una codificación más sofisticada y en este punto, el ciclo empieza nuevamente. Un niño que aprende a diferenciar gatos de perros es un ejemplo concreto de la naturaleza cíclica del cambio cognitivo. Un niño que diferencia gatos de perros sólo en base a su tamaño podrá presentar errores de clasificación.

A través de la incorporación de otras características, tales como diferencias en los sonidos de los animales o las formas de la oreja, el niño aprenderá a diferenciar gatos de perros de manera más minuciosa. Este incremento en el conocimiento, a su vez, le permitirá al niño codificar características adicionales (p.e. la posición de los ojos) permitiéndole incorporar características y normas más sofisticadas.

Sternberg también caracteriza el desarrollo en términos de ciclos continuos y "loops" de retroalimentación. La relación entre el conocimiento base del niño y sus procesos de adquisición de conocimiento (Sternberg, 1984,1985 ) es la mima que la relación entre el conocimiento predictivo del niño y su codificación (Siegler, 1986a).

Según Sternberg, los procesos de adquisición de conocimiento crean un conocimiento-base seguro. Este conocimiento-base, a su vez, permite formas sofisticadas de adquisición y el ciclo continúa. Sternberg propone luego una relación similar entre los procesos metacognitivos del niño y los procesos responsables de la ejecución de las tares. "Loops" de retroalimentación continuos entre los dos tipos de procesos facilitan el desarrollo cognitivo. Sternberg sostiene que los procesos metacognitivos responsables del planeamiento y la evaluación dan información relevante a los procesos responsables de la ejecución de las tareas. La retroalimentación desde los procesos de desempeño hacia los procesos metacognitivos sobre el éxito o fracaso en la ejecución de la tarea, conlleva a la mejora en el planeamiento 
y la evaluación. Esta mejora en el planeamiento da como resultado una mejora en el desempeño y el ciclo continúa.

Los principios organizacionales del desarrollo cognitivo: estadios, ramas (ramificaciones), y "loops" de retroalimentación, permiten visualizar y comunicar la naturaleza del desarrollo cognitivo de la manera más fácil y eficiente posible. En general, el éxito de los estadios depende del nivel de análisis empleado. En ocasiones niveles relativamente globales de análisis se ajustan más a las características de los niños (Case, 1985), mientras que en otras, niveles moleculares resultan mejores (Siegler, 1986b).

El movimiento actual en la psicología del desarrollo cognitivo, tiende a alejarse de las teorías de estadios, y propone modelos de desarrollo ramificados o de "loops" de retroalimentación dado el cuestionamiento respecto a sus limitaciones.

\section{Los procesos cognitivos}

La comprensión del desarrollo cognitivo requiere la especificación de los procesos o mecanismos a través de los cuales se desarrolla el pensamiento de los niños. Los psicólogos del procesamiento de la información han reconocido tradicionalmente dos tipos de procesos: los procesos ejecutivos y los procesos no-ejecutivos.

Los procesos ejecutivos, señalados en la literatura del desarrollo por Vygotsky, respecto a la función de planeamiento del lenguaje, son aquellos que están comprometidos en el planeamiento, monitoreo y evaluación del desempeño en tareas intelectuales. Los términos "metacognitivo" y "ejecutivo" son a veces utilizados de manera indistinta.

Por otro lado, los procesos no-ejecutivos son aquellos comprometidos en el desempeño en tareas de solución de problemas. Un modelo de desarrollo cognitivo completo, necesita de ambos tipos de procesos. La Tabla 1 resume la posición de cada teórico en función a los procesos ejecutivos y no-ejecutivos, según su posición respecto a los otros factores analizados. 


\section{Los procesos ejecutivos}

Los procesos ejecutivos propuestos por los teóricos actuales en relación al desarrollo cognitivo difieren de un modelo a otro, a pesar de que dentro de cada modelo los procesos presentan funciones similares, dado que guían las actividades de solución de problemas y actividades intelectuales en el planeamiento, monitoreo y evaluación del desempeño en tareas cognitivas.

Robbie Case $(1984,1985)$ señala que la habilidad de los niños en lograr estructuras de control ejecutivo sofisticadas se basa en gran parte en el cambio cognitivo. Según Case, las estructuras de control ejecutivo consisten en tres partes interrelacionadas: una representación de la situación problema, una representación del objetivo del problema y una representación de la estrategia del problema. Un niño que no conserva la estructura de control ejecutivo para tareas de conservación de líquido, podrá abarcar la siguiente representación : (a) al representar la situación problema, el niño notará la presencia de dos vasos de precipitación de diferente tamaño de agua de color; (b) en la representación del objetivo del problema, el niño se dará cuenta que debe decidir cuál recipiente contiene más agua y (c) en la representación de la estrategia del problema o procedimiento deberá decidir escoger el vaso de precipitación con más alto nivel de agua. Esta estructura de control ejecutiva ilustra la forma de pensamiento característica de un niño en el primer subestadio de nivel cognitivo. Las estructuras de control de un niño del segundo, tercer o cuarto subestadio contienen, respectivamente dos, tres o cuatro unidades para cada tipo de representación.

Case propone que las transiciones entre estadios son el resultado de re-representaciones de problemas, o de la integración jerárquica de estructuras de control ejecutivas. Es decir, problemas que originalmente requerían de cuatro unidades representacionales, requerirán más adelante de una sola unidad del siguiente estadio cognitivo. Case sostiene que los niños nacen con un conjunto de capacidades de procesamiento general que les permiten ponerse objetivos y construir, evaluar y practicar estrategias de resolución de problemas diseñadas para lograr metas específicas. Estas 
capacidades innatas de procesamiento contribuyen substancialmente al cambio cognitivo, a pesar que su uso sea muchas veces limitado por una capacidad de segundo tipo: la capacidad de almacenamiento a corto plazo. Si el niño posee la capacidad de memoria requerida para la integración jerárquica, se da la transición entre estadios, sino el desarrollo cognitivo deberá esperar los cambios que son prerrequisito a nivel de memoria a corto plazo.

Case propone que el incremento en la capacidad de memoria a corto plazo no se debe a la capacidad de memoria, sino al incremento de la eficiencia operacional en la capacidad de memoria. Por lo tanto, a pesar que el tamaño de la capacidad de almacenamiento total no se expanda con la edad, la proporción del espacio total que se dedica a resolver problemas particulares disminuye a medida que crece en edad.

Según Case, la eficiencia operacional resulta de (a) la automatización o el incremento de la eficiencia en el procesamiento producida por la práctica extensa y (b) maduración biológica, particularmente mielinización neurológica.

En resumen, Case sostiene que los progresos en el desarrollo cognitivo se dan a medida que las estructuras de control ejecutivas se integran jerárquicamente a través de las capacidades de procesamiento innatas del niño y especialmente a través del incremento en la eficiencia operacional de su capacidad de almacenamiento a corto plazo. Sus ideas sobre la mielinización, a pesar de que son posibles, son altamente especulativas y su propuesta acerca de que los niños nacen con determinadas capacidades de procesamiento, también pueden ser debatidas. Sin embargo, su modelo de desarrollo cognitivo ha generado numerosas observaciones empíricas y al análisis del desarrollo intelectual de los niños y ha brindado un espacio para las aplicaciones educativas (Siegler, 1986b). Su nivel de análisis global junto con su propuesta de las unidades de pensamiento en el niño tiene implicancias importantes para la instrucción en los salones de clase.

La teoría de Case resalta la utilidad de ayudar al niño a aprender a través de enseñarle a (a) especificar la naturaleza de la situación problema, 
(b) establecer objetivos realistas frente al problema y (c) formular estrategias efectivas para la resolución de los problemas. Por ejemplo, a los niños se les puede enseñar a resolver problemas de multiplicación poco convencionales (p.e. $3 \mathrm{x}_{-}=12$ más que $3 \times 4=\_$) ayudándoles a identificar $\mathrm{y}$ practicar las estrategias más eficientes de completamiento de tareas y eliminando el uso de estratégicas comparativamente ineficientes. Las aplicaciones educacionales incrementan el valor de los modelos de desarrollo cognitivo y por lo tanto, el modelo de Case aún mantiene la atención de psicólogos educacionales del desarrollo cognitivo.

Robert Sternberg $(1984,1985)$ por su parte, propone un modelo de la inteligencia y del desarrollo intelectual en base a tres tipos de componentes o unidades de procesamiento de la información: metacomponentes, componentes de desempeño y componentes de adquisición del conocimiento. De estos tres componentes, sólo los metacomponentes satisfacen las funciones de procesamiento ejecutivo.

Sternberg (1984) identifica nueve tipos de procesamiento metacomponencial. Un niño que completa una tarea intelectual deberá:

(a) determinar la naturaleza del problema,

(b) seleccionar los componentes de desempeño apropiados para resolver el problema y

(c) seleccionar una estrategia para combinar los componentes de desempeño de manera óptima.

(d) Luego deberá seleccionar una o más representaciones de la información importante para la solución del problema y, dado un tiempo limitado para la solución del problema,

(e) decidir cómo manejar sus recursos de procesamiento. Finalmente, el niño deberá

(f) monitorear su desempeño,

(g) interpretar la retroalimentación sobre la efectividad de sus esfuerzos,

h) decidir cómo actuar en función a la retroalimentación positiva o negativa, $y$

i) modificar su desempeño en respuesta a la retroalimentación. 
Según Sternberg, el desarrollo cognitivo depende sobretodo de los metacomponentes. Los metacomponentes, capaces de monitorear su propia efectividad, pueden aprender de sus errores y éxitos, y por lo tanto, modificar continuamente su propio funcionamiento. También los "loops" de retroalimentación hipotéticos entre el desempeño, la adquisición de conocimiento y los metacomponentes, proveen información adicional, facilitando el desempeño o logro y el desarrollo intelectual.

El modelo de Sternberg de cambio cognitivo, especialmente su énfasis en el procesamiento metacomponencial, explica muchos de los hallazgos en la literatura del desarrollo cognitivo. Numerosos estudios, por ejemplo, concluyen que los niños crecen hasta ser mayores y mientras se vuelven "expertos", invierten relativamente más tiempo planeando las soluciones a los problemas y menos tiempo en la solución misma del problema. Por lo tanto, los niños mayores, cognitivamente más avanzados, dedican relativamente más tiempo al procesamiento ejecutivo que los menores, cognitivamente menos avanzados.

Por otro lado, una limitación de la teoría de Sternberg es que el autor basa su teoría en estudios realizados anteriormente más que en estudios diseñados para evaluar sus propias predicciones. Otra dificultad con el modelo de Sternberg es que sus metacomponentes, si bien son importantes para el desarrollo intelectual son difíciles de definir operacionalmente, siendo su utilidad empírica difícil de evaluar.

Robert Siegler sostiene, al igual que Sternberg y Case, la importancia de la construcción de estrategias en el desarrollo cognitivo. A través del análisis de las estrategias elegidas por los nifios para resolver problemas aritméticos (Siegler, 1986b), concluye que el desempeño y el desarrollo cognitivo mejoran en la medida en que los niños utilizan estrategias "recuperadas" y menos las de acompañamiento, tales como conteo o el uso de sus dedos, como apoyos para la resolución de problemas. Siegler otorga también importancia a la práctica de asociar determinados problemas aritméticos con una gama de alternativas de respuesta. El sostiene que las asociaciones preexistentes, los números particulares en los problemas aritméticos y la frecuencia de la exposición a cada problema, conlleva a una 
mayor fuerza de asociación. A pesar de que el modelo de asociación de Siegler aún necesita más trabajo, su aproximación parece apropiada para el desarrollo de nuevas predicciones sobre el rol de las estrategias de construcción en el desarrollo cognitivo, así como en las unidades de procesamiento ejecutivo de los niños en diferentes áreas.

En términos generales, las estrategias juegan roles importantes en los modelos de Case, Sternberg y Siegler, sin embargo, los sistemas dentro de los cuales operan las estrategias difieren en las tres teorías.

\section{Los procesos no-ejecutivos}

Sternberg y Siegler señalan la importancia de los procesos no-ejecutivos en el desarrollo del pensamiento de los niños. Los componentes de desempeño no-ejecutivos de Sternberg (1985) incluyen los componentes de codificación, combinación y comparación. Según Sternberg, la información es organizada y comparada con información antigua a través de los componentes de combinación y comparación, y finalmente, la respuesta a un problema dado o tarea la proporciona el componente de respuesta. A pesar que Sternberg seńala que los procesamientos metacomponenciales ejercen una influencia mayor y más importante en el desarrollo del procesamiento no-ejecutivo, también considera que la automatización de los procesos ejecutivos y no-ejecutivos producen algún cambio cognitivo. A medida que los niños ganan experiencia con cierto tipo de problemas y tareas, sus procesos son menos controlados y más automáticos, mejorando su desempeño y facilitando su desarrollo intelectual.

$\mathrm{Al}$ argumentar que la codificación influye directamente en el aprendizaje, Siegler (1986a) también enfatiza su rol en la producción del cambio cognitivo. Según Siegler, la codificación efectiva de información en los niños, les permite construir reglar más elaboradas para operar en su entorno. Siegler define la codificación de manera más amplia que Sternberg, utilizando el término para referirse a la interpretación y organización así como a la percepción y almacenamiento de la información, y propone una serie de formas en las cuales los procesos de codificación y combinación 
conllevan al desarrollo cognitivo. Tratando de relacionar el desarrollo psicológico con la evolución biológica Siegler sugiere que la presión de selección conlleva a los niños hacia funcionamientos cognitivos más sofisticados (Siegler, 1984) y que los cambios en la codificación del niño sobre los aspectos de su ambiente, producen también variaciones cognitivas.

Si bien los resultados de investigaciones en la psicología del desarrollo cognitivo justifican la importancia de la codificación y de los procesos relacionados con ésta, ni Sternberg ni Siegler han logrado explicar la manera en que la codificación se desarrolla. Numerosos estudios indican que los niños se vuelven más minuciosos en el procesamiento de la información a medida que crecen. En otras palabras, con el incremento en la edad los niños parecen codificar y tomar en cuenta cada vez más la información que está disponible en su entorno en relación a un problema dado. A través de la inclusión de codificación y de los procesos de combinación en sus modelos de desarrollo intelectual, Sternberg y Siegler sostienen la importancia de la misma.

Siegler (1986a) se centra en la codificación como su propio modelo de desarrollo, a pesar que reconoce el rol de la automatización en la producción del cambio cognitivo. Siegler también reconoce el rol de la generalización en el desarrollo cognitivo efectivo, y en este nivel sus propuestas reflejan también las de Klahr.

Para Klahr (1984) el pensamiento se desarrolla a medida que se generaliza la información y la experiencia, detectando regularidades y eliminando las redundancias en los procesos. Klahr estudia el pensamiento del niño a través del análisis de su procesamiento y desempeño en tareas de conservación de número de Piaget.

Klahr (1984) sostiene que la experiencia del niño es registrada en una línea de tiempo interna. En las tareas de conservación de número, por ejemplo, la línea de tiempo del niño puede incorporar la siguiente información: el niño ve un fila de bolitas, las cuenta y determina que hay seis bolitas en la fila. Luego el niño ve a otra persona que junta las bolitas, las cuenta de nuevo y determina una vez más que hay seis en la fila. 
Habilidades cognitivas

Para Klahr, el niño desarrolla intelectualmente a través de:

(a) la generalización de la información o la detección de regularidades en las experiencias registradas en su línea de tiempo,

(b) haciendo más eficiente el procesamiento de la información a través de la eliminación de pasos redundantes en la línea de tiempo y

(c) elaborando clases transformacionales.

La detección de regularidades en las experiencias de conservación de número puede tomar tres formas: el niño puede generalizar a través de los objetos, a través de los símbolos cuantitativos y sobre las transformaciones. El proceso de eliminación de redundancias mejora la eficiencia a través de localizar y luego remover los pasos innecesarios. Por lo tanto, en las tareas de conservación del número, un niño eventualmente para de contar las bolitas, en función de la compresión o expansión, dándose cuenta que tales procedimientos mantienen el número de bolitas en la fila sin cambiar. Finalmente, el niño elabora su conocimiento de transformación de clases, adquiriendo la habilidad de la conservación.

Para explicar la naturaleza del desarrollo de destrezas tales como la conservación, Klahr construye sistemas de producción auto-modificables, o simulaciones computarizadas capaces de desarrollar en respuesta a nuevas experiencias. Pocos sistemas de producción de auto-modificación han sido desarrollados en la actualidad.

Sin embargo, el modelo de Klahr, al igual que el de Sternberg parece ser útil para explicar los resultados de investigaciones anteriores y para generar nuevas predicciones (Siegler, 1986a). La mayor contribución de la aproximación de Klahr es el de las simulaciones auto-modificables por computadora. Su concepción de generalización representa uno de los mecanismos de cambio intelectual más precisos que han sido propuestos hasta la fecha (Siegler, 1986a).

Finalmente, así como Klahr, Kurt Fischer (1980) también sostiene que la generalización juega un rol en la producción del cambio cognitivo, sin embargo, donde Klahr asigna un rol preponderante a los procesos de 
generalización, Fischer asigna un menor rol a los mecanismos. Fischer propone cinco reglas de transformación, cuatro de ellas (sustitución, enfoque, composición y discriminación) especifican la forma en que los niños desarrollan dentro de un nivel dado, y la quinta regla (intercoordinación) señala la manera en que los niños progresan desde un nivel de habilidad hacia el otro.

Fischer señala que el proceso de sustitución o de generalización de una habilidad lograda en el contexto de una tarea particular hacia otra muy parecida, conlleva al desarrollo dentro del mismo nivel. Un ejemplo del juego en los niños ilustra este proceso. Un niño originalmente haciendo que una muńeca doctor interactúa con una paciente femenina aplica esta misma destreza en la medida que hace que la muñeca doctor interactúe con un paciente masculino, el nińo sustituye al paciente masculino por la paciente femenina. A través del proceso de enfoque, el niño combina dos destrezas relacionadas utilizándolas en sucesión.

Por ejemplo: (a) primero un niño hace que una muñeca doctor interactúe con una muñeca paciente y luego cambia a la interacción entre una muñeca enfermera y una muñeca paciente, dejando a la muńeca doctor fuera del episodio. (b) Segundo, a través de la combinación de habilidades relacionadas, un niño combina dos habilidades similares para formar una habilidad única, más compleja. (c) Tercero, a través de la combinación, un niño hace que una muñeca enfermera y doctor juntas examinen a la muñeca paciente. (d) Cuarto, Fischer señala que el proceso de diferenciación conlleva al desarrollo dentro del nivel, en este sentido los mecanismos de desarrollo de Fischer se superponen con los mecanismos de Werner y los de Klahr.

Según Fischer (1980), el proceso de diferenciación se acompaña de otras normas de transformación y supone la separación de una habilidad en sus distintos componentes de tal manera que cada componente esté mejor definido. A través de la diferenciación el niño demuestra que la destreza combinada de hacer que una muñeca doctor y una muñeca enfermera examinen juntas a la muñeca paciente separa su interacción de 
tres direcciones en su constituyente interacción de dos direcciones (interacciones entre doctor/paciente, enfermera/paciente y doctor/enfermera), resultando en una mayor claridad y elaboración de los roles de estas interacciones constituyentes.

Finalmente, Fisher propone que los procesos de intercoordinación conllevan al niño que se encuentra funcionando en un nivel de habilidad, al siguiente nivel cognitivo. Sin embargo la descripción de la forma en que la intercoordinación funciona parece ser algo vaga, lo cual limita su utilidad empírica.

Los diferentes mecanismos de desarrollo cognitivos propuestos por Case, Sternberg, Siegler, Klahr y Fischer nos mmuestran que el consenso entre las cooncepciones de los mecanismos de de desarrollo entre los psicólogos aún no se ha logrado pese a que se han desarrollado numerosas investigaciones desde las propuestas de Piaget, Vygotsky y Werner. Los mecanismos del desarrollo en la actualidad incorporan diferencias importantes entre dos tipos de procesamiento: ejecutivo y no ejecutivo, y en algunos modelos, especialmente en el de Klahr, se ha realizado un progreso importantes en especificar la manera en que los mecanismos de desarrollo operan.

\section{El conocimiento}

Los teóricos del desarrollo cognitivo sostienen que el conocimiento juega un rol importante, aunque suplementario en el desarrollo cognitivo. En sus propios modelos de desarrollo, Klahr, Siegler, Case y Sternberg se concentran en tratar de explicar los mecanismos responsables del cambio intelectual.

Klahr (1984) señala que la comprensión de los procesos de transición requiere la consideración de tipos de información particular que se encuentran en el procesamiento de la información.. Su propuesta de mecanismos generalizados se aplica en la adquisición de las habilidades conservación, pero no necesariamente en la adquisición de otro tipo de habilidades. 
Siegler (1986a) señala que el conocimiento que traen los niños les ayuda a determinar los conceptos que deben codificar o las estrategias que deben construir en situaciones particulares.

Case (1985) conceptualiza las estructuras de control ejecutivo como conocimiento de procedimiento, y asume que los avances en el conocimiento procedimental y declarativo dan pie al avance en el otro, aunque no ha podido probarlo empíricamente.

En comparación a Klahr, Siegler y Case, Sternberg (1985) amplía el rol del conocimiento en su modelo de desarrollo. El asegura que el niño en la medida que desarrolla su base de conocimiento contribuye con su desarrollo intelectual y propone tres componentes de adquisición de conocimiento diseñados para indicar el incremento en la base de conocimiento. Primero, el niño debe codificar de manera selectiva la información que le llega, discriminando la información relevante de la irrelevante y permitiendo sólo el procesamiento de la información relevante. Segundo, el niño debe combinar selectivamente la información codificada, formando e integrando un todo interpretable. Tercero, el niño debe comparar selectivamente la información codificada y combinada con la información antigua ya guardada en memoria, estableciendo relaciones entre las adquisiciones nuevas $\mathrm{y}$ antiguas.

Por ejemplo, cuando se lee una novela de misterio, el niño intenta resolver el misterio discriminando entre las claves relevantes, combinándolas de manera significativa para llegar a los sospechosos, y relacionando la información incriminatoria de los sujetos al conocimiento previo sobre los tipos de personajes y situaciones relatadas en la novela.

Según Sternberg, el conocimiento previo del niño lo gúa en los primeros dos procesos así como en el tercero. Tanto las comparaciones que realiza el niño como las claves codificadas y la integración construida dependerán del conocimiento previo de las novelas de misterio.

Sternberg señala que cuando los procesos de codificación selectiva, combinación selectiva y comparación selectiva se extienden a tareas y 
situaciones nuevas, apoyan las adquisiciones de nuevos conocimientos en los niños. Más aún Sternberg sostiene que las habilidades superiores de seleccionar, codificar, combinar y comparar se ejercitan en tareas y situaciones nuevas, y ayudan a diferenciar a los niños intelectualmente talentosos de los no-talentosos (Davidson y Sternberg, 1984). En una serie de experimentos, Davidson y Sternberg (1984) demostraron que la codificación selectiva, combinación y habilidades de comparación superiores diferencian el desempeño de niños talentosos de los no-talentosos en problemas matemáticos. Se demostró también que el proporcionar información relevante así como facilitar la integración apropiada, ayuda a mejorar el desempeño de sujetos no talentosos más que en sujetos talentosos.

El proveer información de comparación selectiva adicional ayuda también a mejorar el desempeño de niños no talentosos más que el de niños talentosos. Estos hallazgos apoyan la propuesta de los investigadores sobre el rol del insight en el talento intelectual.

Son dos las conclusiones más importantes de los procesos de adquisición de conocimiento propuestos por Sternberg: (a) primero, Sternberg ha demostrado que los mecanismos diseñados para explicar el desarrollo intelectual normal pueden ser la base de la inteligencia excepcional y (b) segundo, los procesos de adquisición del conocimiento propuestos tienen aplicaciones educacionales, dado que sugieren que las destrezas de insight de los niños deberán ser facilitadas a través del entrenamiento.

Klahr, Siegler, Case, Sternberg, Keil y Carey analizan los cambios en las estructuras de conocimiento de los niños y en las representaciones más que en los procesos que están a la base del cambio estructural.

Según Keil (1984) la actual dinámica de cambio depende básicamente en la estructura de lo que se conoce y en cómo este conocimiento es utilizado en el mundo. El sostiene que el investigador del desarrollo cognitivo primero debe examinar la estructura del conocimiento de dominio-específico y sólo entonces puede hacer inferencias acerca de cómo ocurren los cambios en este nivel. Klahr aparentemente adopta esta aproximación dado que sus mecanismos de desarrollo explican la manera en la 
que los niños adquieren habilidades de conservación y no explican la manera en que todo el desarrollo cognitivo ocurre.

Keil señala que un conjunto limitado de procesos de aprendizaje generales simples, tales como la diferenciación transforman las estructuras de conocimiento de dominio específico, creando el desarrollo cognitivo. Para demostrar que la naturaleza de la información transformada es más importante que los procesos de transformación en sí mismos, Keil (1984) examina numerosas áreas de la investigación del desarrollo, encontrando que la literatura presenta información consistente en el área de pensamiento del niño respecto a la forma de internalizar los conceptos más allá del concepto en sí mismo.

Por ejemplo, mientras que los niños más pequeños definen "papá" a través de consideraciones de edad y características comportamentales, los niños mayores definen "papá" en función a consideraciones sociales y definiciones preestablecidas. Keil señala que este cambio ocurre en tiempos diferentes para conceptos diferentes, sugiriendo que está determinado básicamente por las estructuras de los conceptos en sí mismos más que por la transición general de un conocimiento general hacia uno más específico.

Carey señala también que la reorganización del conocimiento debe ser explicada en función a uno o más tipos de procesos cognitivos, aunque no indica la forma que estos procesos debieran tener. Carey (1985b) tiende a ser menos explícito que Keil al explicar los posibles mecanismos, pero ofrece una descripción detallada sobre la forma en que los niños aprenden la biología a partir de su propia comprensión de la naturaleza humana y la conducta animal.

Los resultados de las investigaciones de Carey indican que los niños en edad preescolar comparan los objetos animados e inanimados con las personas para determinar si los objetos poseen determinadas propiedades animales. Ella propone una línea de desarrollo del pensamiento de los niños con respecto a los animales y seres vivientes que va desde los 4 años hasta los 10 años, mostrando a través de sus resultados cómo los conceptos de los niños mayores sobre "animal" y "seres vivos" son lo suficientemente 
abstractos como para formar la base de una teoría rudimentaria sobre la biología. Para Carey el desarrollo cognitivo supone la reestructuración de los conocimientos y categorías conceptuales a partir de los cuales se generan nuevas teorías.

El modelo de desarrollo cognitivo de Carey nos brinda un marco teórico para analizar los cambios estructurales en diferentes categorías conceptuales. Su teoría también permite reinterpretar algunos resultados de investigaciones ya realizadas. Por ejemplo, los resultados de Piaget sobre el animismo infantil puede ser analizado desde las investigaciones de Carey sobre las teorías de la biología de los niños, según esta, el animismo infantil no se basa solamente en las capacidades de razonamiento causal de los niños, sino también en su conocimiento sobre la biología (Carey, 1985a).

Los modelos recientes del desarrollo intelectual analizados en esta monografia difieren básicamente en el énfasis que otorgan a los procesos cognitivos y al conocimiento. Sin embargo, los avances en la psicología del desarrollo cognitivo necesitan de una cuidadosa consideración tanto de los procesos como del conocimiento en las teorías del desarrollo intelectual, más aún, las teorías que señalan la interrelación entre los mecanismos del desarrollo y el conocimiento en los niños tienen una valiosa aplicación educativa, que trasciende su utilidad respecto al cambio estructural cognitivo.

\section{Las diferencias individuales}

Los modelos actuales de desarrollo cognitivo deben explicar la manera en que se presentan diferencias individuales entre los ninos se presentan, con el fin de poder explicar mejor el procesamiento de la información y la adquisición del conocimiento.

Case (1985) sostiene que los factores biológicos y experienciales o culturales producen dos tipos de diferencias individuales: Primero, las diferencias en el ritmo de desarrollo de la capacidad de almacenaje a corto plazo y en la eficiencia de las capacidades de procesamiento general, conllevan a diferencias en el ritmo del desarrollo general ejecutivo de los 
niños. Segundo, los niños difieren a nivel del ritmo de su desarrollo ejecutivo en determinadas tareas de dominio específico. Por lo tanto, Case sugiere que el ritmo de desarrollo, mas no el curso del mismo, varía entre los niños.

Por otro lado, Fischer (Fischer y Pipp, 1984) y Klahr (1984) sugieren que el curso del desarrollo puede diferir de un niño a otro, aunque éstos investigadores señalan que los mecanismos a la base del cambio cognitivo son los mismos para todos los niños.

Fischer propone que la adquisición de destrezas siempre supone influencias biológicas y ambientales. Según Fischer, el desarrollo de determinadas habilidades varía de niño a niño pese a que las reglas de transformación se mantienen iguales. De manera similar, $\mathrm{Klahr}$ asume que debido a que las experiencias de los niños difieren, las líneas de tiempo que registran sus experiencias también varían de uno a otro. Cada niño desarrolla a través de la generalización, sin embargo, el curso del desarrollo intelectual de cada niño depende de las experiencias particulares que se mantienen en su línea de tiempo.

Las diferencias individuales señaladas en el modelo de desarrollo cognitivo de Siegler $(1984,1986 a)$ parten de dos fuentes: Primero, la variabilidad de los mecanismos de la evolución producen variación entre los niños, y segundo, las variables motivacionales también conllevan a diferencias en el desempeño. Debido a que los intereses facilitan la atención y la memoria (Renninger y Wozniak, 1985), las diferencias en los intereses de los niños sea en problemas particulares o tareas, puede llevar a diferencias posteriores en su conocimiento sobre estos problemas o tareas. Por lo tanto, un niño que esté interesado en gatos u otro tipo de mascotas, observará animales por un período de tiempo mayor que otros niños y es posible que recuerde mejor las características de los animales que un niño al cual no le interesan.

En los términos de Siegler, el niño interesado puede codificar más características de los gatos que el niño menos interesado, con lo cual logrará un mayor conocimiento y acercamiento a reglas más sofisticadas de la 
conducta animal. Por otro lado, las diferencias en las reacciones de los niños hacia el fracaso puede ocasionar diferencias en el desempeño intelectual. Algunos niños atribuyen el fracaso a su falta de habilidad, mientras otros lo atribuyen a causas externas a ellos mismos (e.g. mala suerte).

Finalmente, si bien el modelo de Sternberg señala la importancia de diversas razones de las diferencias individuales, él pone énfasis en las variaciones de las respuestas de los niños hacia situaciones nuevas (Berg y Sternberg, 1985a). Así como Siegler, Sternberg señala que los estados motivacionales de los niños producen diferencias individuales en el funcionamiento intelectual. Niños que desean experimentar tareas y situaciones nuevas incrementan sus oportunidades de aprendizaje sobre estas tareas o situaciones. Asimismo, las diferencias en las capacidades de los niños para enfrentar la novedad, especialmente sus capacidades para procesar y adquirir información nueva, influencia su desempeño en tareas intelectuales.

Las diferencias en las habilidades de los niños para codificar, combinar y comparar información nueva diferencia a los individuos talentosos de los no talentosos (Davidson y Sternberg, 1984). Por otro lado, las diferencias en las capacidades de los niños para elaborar información nueva de manera espontánea para facilitar la memoria de esta información distingue académicamente a los alumnos exitosos en la primaria de los menos exitosos.

En nuestra opinión, una teoría completa del desarrollo cognitivo debe explicar las diferencias individuales en el desarrollo y en el desempeño. Por otro lado, el potencial de aplicaciones en el campo educativo incrementa significativamente el valor de los modelos de las diferencias individuales. $\mathrm{Si}$ bien los resultados de las investigaciones en la literatura del desarrollo confirman los modelos de Siegler y Sternberg, se necesita investigación adicional para esclarecer y evaluar las diferentes posturas desarrolladas por los teóricos actuales del desarrollo cognitivo.

\section{El contexto}

Vygotsky (1978) señala que el desarrollo intelectual del niño se basa en la internalización de conductas sociales y sistemas simbólicos 
culturalmente producidos. Numerosos teóricos en la actualidad incorporan también factores de contexto en sus modelos de desarrollo, considerando no sólo al niño sino también el contexto en el cual el cambio cognitivo y el desarrollo intelectual ocurre.

Los factores de contexto que influyen en el desarrollo pueden ser divididos en dos categorías: (A) las experiencias del niño y (b) su ambiente. Las experiencias de los niños, sus actividades diarias y las consecuencias de las mismas, son únicas. Por otro lado, cada niño comparte su ambiente social y cultural con otros niños, específicamente con una determinada subpobla-ción de niños. Así, a un niño le puede encantar leer novelas de misterio, mientras que a otro le puede encantar leer poesía, debido a que ambos niños han tenido experiencias diferentes, pese a compartir un mismo entorno: ambos viven en una sociedad que valora y refuerza la lectura.

Otros niños, que viven en ambientes diferentes, tales como una sociedad que valore las destrezas atléticas y físicas sobre las habilidades intelectuales, presentarán habilidades en deportes diferentes.

En términos generales, los modelos de desarrollo cognitivo recientes no se han mostrado particularmente interesados en las consideraciones de contexto, pese a que algunos han reconocido su importancia.

\section{La experiencia}

Klahr (1984) y Sternberg (1985) seńalan el impacto de las experiencias en el desarrollo intelectual del niño. Tal como se señaló anteriormente, Klahr sostiene que los niños se desarrollan a través de la generalización de las experiencias grabadas en sus líneas de tiempo.

Sternberg incorpora las experiencias de los niños en su modelo de desarrollo cognitivo. de manera más compleja que Klahr. La Teoría Triárquica de la Inteligencia Humana de Sternberg (1985), supone tres subteorías: 
(a) una teoría componencial que especifica la manera en que el desempeño de los metacomponentes y los procesos de adquisición de información operan,

(b) una subteoría contextual que relaciona la vida mental del individuo con su ambiente, $y$

(c) una subteoría experiencial que relaciona la vida mental del individuo con sus experiencias.

En su subteoría experiencial Sternberg propone que el procesamiento de información opera a lo largo de un continuum experiencial. En un límite del continuum el individuo no tiene experiencia alguna con determinadas tareas y situaciones, las tareas y situaciones son completamente novedosas, mientras que en el otro extremo del continuum el individuo presenta el procesamiento de la información completamente automatizado.

Sternberg (1985) sostiene que el procesamiento automático de la información conlleva al crecimiento cognitivo, por lo tanto, las experiencias del niño juegan un rol importante en su desarrollo. Un niño que lee novelas de misterio podrá aprender a codificar y combinar claves relevantes de manera más rápida y efectiva que un niño que lee poesía. Un lector de poesía, por otro lado, podrá aprender a interpretar simbolismos a través de la comparación de los símbolos en los poemas de manera más rápida que un lector de novelas de misterio.

La concepción de Sternberg de un continuum experiencial a través del cual se desarrolla el procesamiento de la información tiene implicancias importantes para la evaluación y medición intelectual. Sternberg sostiene que las diferencias en las habilidades de los niños tienen relación con la novedad y automatización del procesamiento de información, diferenciando de esta manera a los niños más inteligentes de los menos inteligentes. Por lo tanto, las pruebas de inteligencia deberían incorporar tareas de desempeño relativamente novedosas o que estén en el proceso de ser automatizadas. A veces tareas diferentes son necesarias para evaluar las respuestas de los niños frente a la novedad y su automatización de los procesos ejecutivos y no ejecutivos. 


\section{El ambiente}

En su subteoría contextual de la inteligencia, Sternberg relaciona el procesamiento de la información con los ambientes particulares en los que éste ocurre. El señala que el funcionamiento intelectual es una actividad mental dirigida hacia la adaptación y la selección y moldeamiento de ambientes reales relevantes a la propia vida (Sternberg, 1985).

Por lo tanto, ambientes diferentes producen necesidades adaptativas diferentes y formas de actividad mental diferentes. Por ejemplo, la actividad mental esencial para una adaptación exitosa a la escolaridad difiere substancialmente de la actividad necesaria para la adaptación en el hogar. Mientras los profesores valoran una prosa clara y concisa, los padres y hermanos refuerzan historias verbales e imaginativas. Muchas veces no son las habilidades intelectuales en sí mismas, sino su importancia relativa la que difiere de un ambiente a otro.

Las influencias ambientales son importantes no sólo cuando se considera el nivel de inteligencia del niño, sino también cuando se considera su desarrollo intelectual. Los ambientes en los cuales los individuos deben adaptarse cambian a medida que los individuos crecen. Los niños se deben adaptar al hogar y la escuela, los adultos jóvenes se deben adaptar a nuevas carreras y a nuevos tipos de relaciones (p.e. matrimonio, parejas), y los adultos tardíos se deben adaptar al retiro y posiblemente a la pérdida de sus parejas y amigos. Las condiciones de cambio a las que los individuos se deben adaptar a lo largo de sus vidas son desarrolladas por Erikson (1968) en su teorías de desarrollo psicosocial. Los teóricos contextuales como Sternberg señalan que la actividad mental varía junto con los factores psicosociales y ambientales, debiendo el individuo adaptarse a diferentes circunstancias en diferentes períodos de su ciclo de vida.

Sternberg (1985) señala que las influencias sociales y culturales juegan un rol crucial en la cognición y por lo tanto la inclusión de este factor en los modelos de inteligencia y desarrollo intelectual es necesario. Si bien las consideraciones ambientales son necesarias, las aproximaciones de contexto en las habilidades humanas y en el cambio cognitivo son en general vagas. 
La subteoría contextual de Sternberg es más prescriptiva que descriptiva, dado que provee un marco teórico para el estudio del desarrollo cognitivo en base a un marco empírico y teórico. Sin embargo, no provee de descripciones detalladas de los factores contextuales relevantes para comprender la actividad mental en diferentes poblaciones, ni provee explicaciones de la forma en que los factores contextuales específicos guían la cognición. Más aún, el cuestionamiento de su utilidad empírica, representa una limitación relacionada a los marcos contextuales como el de Sternberg. A no ser que se desarrollen predicciones específicas y operacionales, los modelos contextuales no podrán ser falseados a través de la investigación.

Sternberg pone énfasis en la adaptación al igual que Piaget. Ambos teóricos señalan que la inteligencia busca la adaptación al ambiente. Por otro lado, otros teóricos actuales consideran a los factores de contexto en el desarrollo sin considerar la adaptación como el fin último para su influencia.

Fischer, por ejemplo, señala que las habilidades de los niños reflejan su ambiente, así como sus características biológicas inherentes. Sin embargo, la instrucción y el soporte social representan dos factores ambientales que pueden variar de sociedad a sociedad y de cultura a cultura.

Case (1985) señala que la cultura juega un rol importante en la transición entre los estadios y en las habilidades logradas en los estadios sucesivos, en la medida que se van logrando niveles superiores.

Finalmente Siegler (1986ayb) señala tres formas en las que el ambiente del niño ayuda a determinar la naturaleza de su actividad mental, dado que el ambiente influye en las habilidades específicas que los niños logran, en los métodos de aprendizaje y en sus motivaciones para el pensamiento y aprendizaje. Los resultados de sus investigaciones apoyan sus propuestas.

En términos generales es importante que las teorías del desarrollo cognitivo incorporen las influencias de contexto en el diseño metodológico de las investigaciones sobre el desarrollo cognitivo del niño, para poder 
explicar los factores que afectan su desarrollo y la forma en que éstos pueden o no alterar la actividad mental, para luego poder generar predicciones específicas que sean probadas empíricamente.

\section{Las limitaciones en el desarrollo}

La idea de las limitaciones en el desarrollo se origina del trabajo de Keil (1981), quien utiliza por primera vez el término para referirse a las limitaciones que se presentaban en el pensamiento de los niños debido a contenidos de dominio determinados.

Los modelos recientes del desarrollo intelectual sugieren dos categorías generales de limitaciones: organísmicas y en relación al dominio. Las limitaciones organísmicas se refieren a las limitaciones relacionadas con las características biológicas inherentes a cada niño, dentro de las que se consideran dos tipos: las relacionadas con la edad y las cognitivas. Por otro lado, las limitaciones relacionadas con el dominio se refieren a las limitaciones impuestas por el contenido particular de las áreas que el niño se espera domine.

\section{Limitaciones organismicas}

Los teóricos de los estadios que relacionan las habilidades cognitivas de los niños con rangos de edad específicos, señalan limitaciones relacionadas con la edad en el desarrollo. Un niño de una edad determinada enfrenta limitaciones en el procesamiento que limitan sus capacidades cognitivas. Las limitaciones disminuyen o se incrementan a medida que el niño crece, produciendo los correspondientes incrementos en sus capacidades.

Piaget, Vygotsky, Case y Fischer, relacionan las limitaciones en el procesamiento con la edad cronológica y proponen que estas limitaciones relacionadas con la edad limitan el ritmo y la naturaleza del desarrollo desde la infancia hasta la adolescencia. 
Para Case, las limitaciones cognitivas están a la base de las limitaciones relacionadas con la edad. Case señala que la eficiencia operacional de la capacidad de almacenamiento a corto plazo del niño, crea limitaciones en el procesamiento relacionadas con la edad. A medida que el niño crece, su memoria a corto plazo se vuelve más eficiente, permitiéndole pensar y aprender de modos más sofisticados.

De manera similar, Sternberg (Davidson y Sternberg, 1985) señala que la capacidad funcional de la memoria de trabajo del niño media la competencia intelectual del niño y, por lo tanto su desempeño. Sin embargo, a diferencia de Case, Sternberg no relaciona esta limitación a la edad cronológica del niño. Finalmente, para Fischer, las limitaciones relacionadas con la edad reflejan el nivel óptimo del niño, o el límite superior (techo) en los tipos de habilidades que el niño de una edad determinada puede construir.

La literatura del desarrollo apoya la idea de limitaciones organísmicas en el desarrollo de dos formas: Primero, el ritmo y la naturaleza del desarrollo cognitivo durante la adultez no es tan impresionante como el ritmo y la naturaleza del cambio intelectual durante la infancia y la adolescencia (Flavell, 1984), resaltando el valor heurístico de algún tipo de limitación relacionada con la edad. Case y Fischer claramente diferencian el desarrollo cognitivo en la niñez del desarrollo en la adultez. Otros teóricos actuales, sin embargo no logran realizar esta diferenciación, a pesar que la diferenciación está probada en la literatura del desarrollo. Segundo, hay evidencia que indica que la capacidad funcional (no necesariamente el tamaño) de la memoria a corto plazo, se incrementa con la edad (Case, 1984; Pascual-Leone, 1970). También existe evidencia que señala que la capacidad funcional de la memoria de trabajo media el desempeño en una amplia variedad de tareas (Brainerd, 1981)

En general, la literatura sobre el desarrollo cognitivo revela que existen limitaciones de memoria en el desarrollo y la cognición, justificando su inclusión en los modelos de desarrollo cognitivo. 


\section{Limitaciones en relación al dominio}

Los teóricos del conocimiento ponen más énfasis en las limitaciones relacionadas con el dominio, que los teóricos de los estadios. Sternberg, por ejemplo señala que la novedad introduce limitaciones relacionadas con el dominio, por lo tanto un niño hábil en resolver determinado tipo de problemas aritméticos puede fallar en comprender las fracciones, la primera vez que debe enfrentarse a éstas. Con la práctica, el procesamiento de la información del niño se automatiza, y las limitaciones en relación al dominio creadas por la novedad desaparecen.

Para Carey (1985b), el conocimiento limitado en determinados dominios, interfiere con la habilidad cognitiva y su desarrollo. Sin embargo, el crecimiento cognitivo se da en la medida que el niño es capaz de sobreponerse a estas limitaciones a través de la adquisición continua de información y la reorganización del conocimiento.

Sternberg y Carey sostienen que el conocimiento limitado y la experiencia limitan el desarrollo en determinados dominios de contenido.

Keil $(1981,1984)$ señala que áreas particulares de contenido limitan la cognición de manera más significativa, dado que percibe al niño como un organismo biológico moldeado por la evolución en busca de dominios de contenido fáciles de lograr y dominar. Según Keil (1984) los niños deben encontrar ciertos dominios como más fáciles de adquirir que otros, maximizando sus habilidades y desarrollando en los dominios naturales, frente a los no naturales. Sin embargo, Keil no logra diferenciar los dominios que los niños encuentran naturales frente a los que no lo son.

En términos generales, los modelos de desarrollo cognitivo propuestos por Case, Fischer, Klahr, Siegler, Sternberg, Keil y Carey son comprensivos pero incompletos, por lo que es necesario continuar investigando y profundizando en los factores que se encuentran a la base del cambio y desarrollo cognitivo. El resumen de los modelos de los siete teóricos del desarrollo cognitivo contemporáneo, en base a los seis factores analizados, se encuentra en el Cuadro 3. 
Cuadro 3

Resumen de posiciones teóricas.

\begin{tabular}{|c|c|c|c|c|c|c|}
\hline TEORLA. & 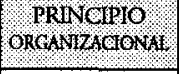 & PROCESOS DE CAMBIO & conocIMINIF & $\begin{array}{l}\text { DIFERENCIAS } \\
\text { nNOM MOUAIES }\end{array}$ & CONTEXTOO & MMUMCIONES \\
\hline CASE & Etapas & $\begin{array}{l}\text { Ejecutivo: Integración jerár- } \\
\text { quica de las estructuras de } \\
\text { control, con eficiencia } \\
\text { operacional en el alma- } \\
\text { cenamiento de la memoria } \\
\text { a corto plazo. }\end{array}$ & $\begin{array}{l}\text { Estructuras de control eje- } \\
\text { cutivo con conocimiento } \\
\text { de procedimientos. }\end{array}$ & $\begin{array}{l}\text { En el rango de de- } \\
\text { sarrollo ejecutivo. }\end{array}$ & $\begin{array}{l}\text { La cultura juega un } \\
\text { rol creciente en las } \\
\text { etapas de transición } \\
\text { mientras el niño se } \\
\text { desarrolla. }\end{array}$ & $\begin{array}{l}\text { Organismicas. Relacio- } \\
\text { nadas con la edad. Efi- } \\
\text { ciencia operacional en } \\
\text { el almacenamiento a } \\
\text { corto plazo. }\end{array}$ \\
\hline FISCHER & Etapas & $\begin{array}{l}\text { No ejecutivo: Dentro de } \\
\text { cuatro etapas de transición. }\end{array}$ & No énfasis particular. & $\begin{array}{l}\text { En el desarrollo de } \\
\text { destrezas específicas. }\end{array}$ & $\begin{array}{l}\text { Influencia ambiental } \\
\text { práctica, instruc- } \\
\text { ción, soporte. }\end{array}$ & $\begin{array}{l}\text { Organísmicas: Relacio- } \\
\text { nadas con la edad. } \\
\text { Nivel optimo de habi- } \\
\text { lidad. }\end{array}$ \\
\hline KLAHR & $\begin{array}{l}\text { Análisis en el } \\
\text { tiempo }\end{array}$ & No ejecutivo & $\begin{array}{l}\text { Procesos de generalización } \\
\text { reflejan dominios de con- } \\
\text { tenido especificos. }\end{array}$ & $\begin{array}{l}\text { Resultado de expe- } \\
\text { riencias diferentes. }\end{array}$ & $\begin{array}{l}\text { Enfasis en el rol de } \\
\text { la experiencia. }\end{array}$ & No considera. \\
\hline SIEGLER & $\begin{array}{l}\text { Ciclos } \\
\text { contínuos }\end{array}$ & $\begin{array}{l}\text { Ejecutivo: Modelo asocia- } \\
\text { do de construcción de es- } \\
\text { trategias }\end{array}$ & $\begin{array}{l}\text { Ayuda a determinar qué } \\
\text { procesos de cambio se } \\
\text { dan. }\end{array}$ & $\begin{array}{l}\text { Mecanismos de va- } \\
\text { riabilidad en analo- } \\
\text { gias, variables de } \\
\text { motivación. }\end{array}$ & $\begin{array}{l}\text { Enfariza el contexto } \\
\text { social en el desarro- } \\
\text { llo. }\end{array}$ & No considera. \\
\hline STERNBERG & $\begin{array}{l}\text { Ciclos contí- } \\
\text { nuos y retroali- } \\
\text { mentación con- } \\
\text { vergente }\end{array}$ & $\begin{array}{l}\text { Ejecutivo: Metacompo- } \\
\text { nentes y retroalimentación } \\
\text { componencial. } \\
\text { No ejecutivo: Componen- } \\
\text { tes de desempeño; automa- } \\
\text { tización. }\end{array}$ & $\begin{array}{l}\text { Componentes de adqui- } \\
\text { sición de conocimiento. } \\
\text { Aún en desarrollo. }\end{array}$ & $\begin{array}{l}\text { Diferentes fuentes, } \\
\text { especialmente de las } \\
\text { respuestas ante si- } \\
\text { tuaciones nuevas. }\end{array}$ & $\begin{array}{l}\text { Contínuo expe- } \\
\text { riencial de la nove- } \\
\text { dad a la automa- } \\
\text { tización. Sub-teoría } \\
\text { contextual que seńa- } \\
\text { la la adaptación al } \\
\text { ambiente. }\end{array}$ & $\begin{array}{l}\text { Organísmica: Capaci- } \\
\text { dad funcional de la } \\
\text { memoria de trabajo. } \\
\text { Relacionadas con el } \\
\text { dominio específico: } \\
\text { novedad. }\end{array}$ \\
\hline KEIL & $\begin{array}{l}\text { Depende de do- } \\
\text { minio de conte- } \\
\text { nido bajo consi- } \\
\text { deración }\end{array}$ & $\begin{array}{l}\text { No específico: Procedi- } \\
\text { mientos de aprendizaje sim- } \\
\text { ples y generales. }\end{array}$ & $\begin{array}{l}\text { Muy importante, la diná- } \\
\text { mica de cambio depende } \\
\text { de la estructura de cono- } \\
\text { cimiento. }\end{array}$ & No considera. & No considera. & $\begin{array}{l}\text { Relacionadas con el do- } \\
\text { minio específico: algu- } \\
\text { nos dominios: de con- } \\
\text { tenido son naturales } y \\
\text { otros adquiridos. }\end{array}$ \\
\hline CAREY & Ramificaciones & No considera. & $\begin{array}{l}\text { Muy importante, enfatiza } \\
\text { la adquisición y reorgani- } \\
\text { zación del nuevo conoci- } \\
\text { miento. }\end{array}$ & No considera. & No considera. & $\begin{array}{l}\text { Relacionados con el } \\
\text { dominio específico: } \\
\text { Conocimiento limita- } \\
\text { do. }\end{array}$ \\
\hline
\end{tabular}




\section{El modelo heurístico de Sincoff y Sternberg}

Para muchos autores el problema del desarrollo cognitivo es muy complejo, y sugieren que una teoría de desarrollo cognitivo debe considerar no sólo los seis factores discutidos en el capítulo anterior, sino también factores relacionados con la motivación y la cognición social (Flavell, 1984, 1985). En este sentido, Sincoff y Sternberg (1989) proponen un modelo heurístico para integrar los factores que deben ser considerados en el análisis del desarrollo cognitivo.

Según el modelo heurístico de Sincoff y Sternberg (1989), las interacciones entre el procesamiento de la información del niño y su experiencia lo lleva a la adquisición continua y reorganización de su conocimiento. Las experiencias del niño (su participación en los eventos de la vida cotidiana) y el procesamiento de la información (en general su interpretación de los eventos) son de suma importancia en la adquisición del conocimiento y en el crecimiento cognitivo. La base de conocimiento del niño es importante para el cambio cognitivo continuo. El modelo se representa como un diagrama de Venn, enfatizando las interacciones entre el procesamiento de la información, las experiencias y el conocimiento del niño (ver Figura 1).

A niyel del procesamiento de información, el desarrollo intelectual requiere de los procesos ejecutivos y no ejecutivos. A través del planeamiento, monitoreo y evaluación, los procesos metacognitivos del niño orientan sus procesos no ejecutivos, que a su vez permiten la adquisición y reorganización del conocimiento procedimental y declarativo. Las estructuras de conocimiento desarrolladas proveen la base para mejorar el procesamiento metacognitivo, formando un "loop" de retroalimentación importante. A lo largo del desarrollo, la memoria de trabajo limita su influencia y a su vez modifica el procesamiento de la información del niño. Es decir, la capacidad de almacenamiento a corto plazo del niño determina en parte la extensión y naturaleza de su procesamiento, dado que la relación entre las limitaciones de memoria y procesamiento es recíproco. 
Habilidades cognitivas

Figura 1

Modelo heuristico de Sincoff y Sternberg (1989)

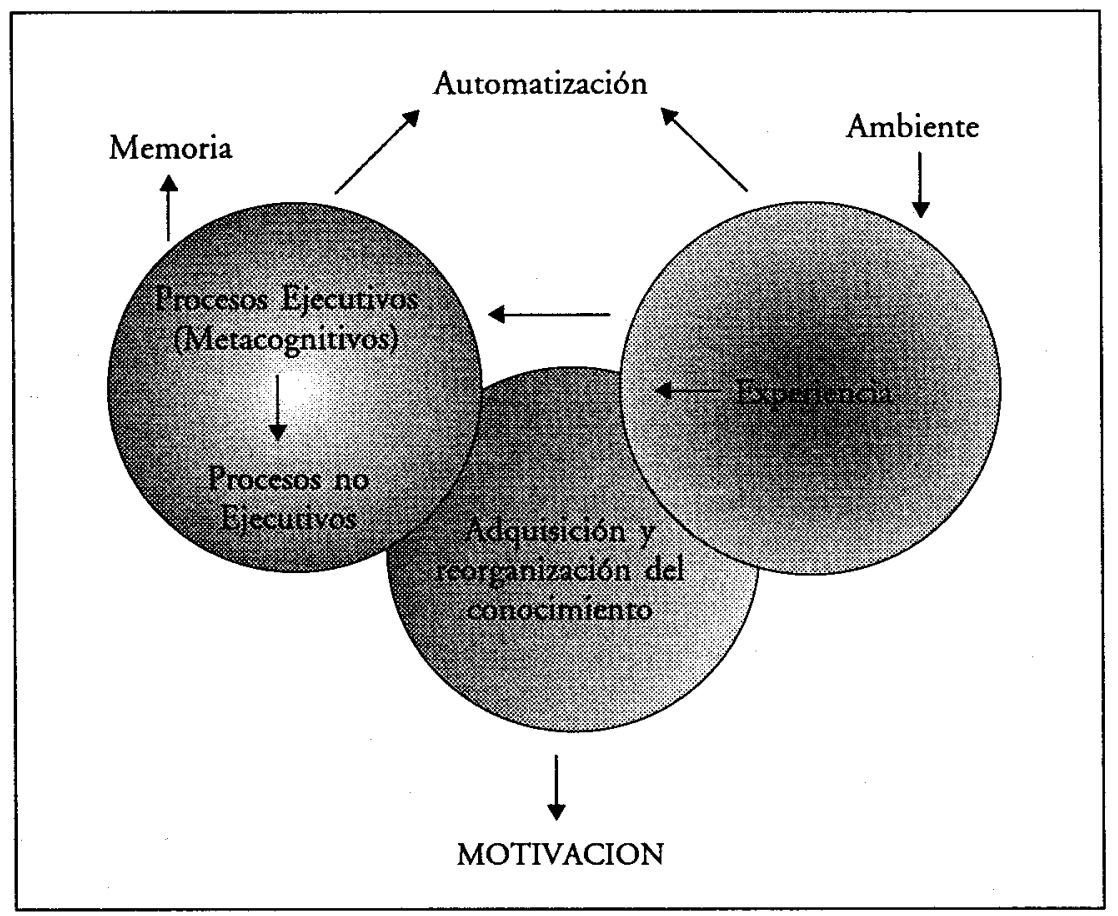

La automatización contribuye a incrementar la eficiencia operacional de la memoria de trabajo y resulta de la interacción entre el procesamiento del niño y sus experiencias repetidas en determinado tipo de problemas. Con la práctica el procesamiento del niño e se vuelve más rápido y eficiente.

A nivel de la experiencia el ambiente cultural y social del niño determina de manera parcial su experiencia, tanto la naturaleza de las experiencias como el valor que el niño les otorga. El niño deberá procesar su experiencia para que éstas puedan influir en la adquisición del conocimiento y en su reorganización. Eventualmente, la automatización del procesamiento puede conllevar a una relación más directa entre la experiencia 
y el conocimiento, una relación (sugerida en el modelo de construcción de estrategias de Siegler) que supone la actualización directa de la información más que un procesamiento más activo.

A nivel de conocimiento, la presencia o ausencia de conocimiento sobre determinado tipo de problema puede influir en su sentimiento sobre los problemas, facilitando o inhibiendo su motivación para (a) buscar experiencias continuas en dominios relevantes y (b) analizar los problemas lo más minuciosamente posible. Si bien Sincoff y Sternberg (1989) proponen una diferenciación de la motivación respecto al procesamiento en su modelo, es necesario investigar la validez psicológica del mismo, dado que Singer y Kolligian (1987) señalan por su parte la imposibilidad de separar los sistemas cognitivos de los afectivos de la personalidad del individuo.

Sincoff y Sternberg (1989) señalan que las interacciones entre las experiencias del niño y el procesamiento de la información dan como resultado el crecimiento cognitivo. El desarrollo del conocimiento procedimental y declarativo no sólo contribuye al mayor crecimiento, sino también refleja el nivel del desarrollo del niño. En el modelo se observan numerosos "loops" de retroalimentación (p.e. los "loops" que abarcan (a) conocimiento y procesamiento y (b) conocimiento, motivación y experiencia), que crean estructuras de conocimiento sofisticadas. El producto del crecimiento cognitivo es la continua ramificación del conocimiento que se da mientras que el conocimiento procedimental y declarativo en diversos dominios es adquirido y organizado.

Los modelos ramificados de crecimiento cognitivo son sustentados en la literatura del desarrollo. Carey señala la emergencia y reorganización del conocimiento biológico y fisico del niño (Carey, 1985b), los estudios psicométricos de las habilidades muestran que las habilidades intelectuales son cada vez más distintas y menos correlacionadas a medida que los nińos crecen, los estudios sobre la cognición social y el desarrollo afectivo de los niños presentan organizaciones ramificadas (Demos, 1974).

Los hallazgos en las investigaciones sobre el desarrollo cognitivo soportan todas las partes del modelo como fuentes importantes de diferencias 
individuales entre los niños. Por ejemplo, las diferencias en las experiencias de los niños (sus antecedentes educacionales, la cantidad de práctica que reciben en diferentes tipos de problemas) crean diferencias en la base de conocimiento que adquieren (Heath, 1983). Asimismo, la experiencia de los niños en el planeamiento metacognitivo conlleva a diferencias en el desempeño frente a las tareas y en la adquisición del conocimiento. Si bien el modelo puede ser utilizado para conceptualizar el desarrollo en diversas áreas, es probable que las limitaciones de orden biológico determinen las áreas en las que este modelo se puede aplicar.

\section{Reflexiones finales}

La investigación y la comprensión sobre el desarrollo cognitivo ha recorrido un largo camino desde que Piaget, Vygotsky y Werner propusieran sus teorías. Sin embargo, quedan aún numerosos cuestionamientos por explorar. Un tema especialmente interesante lo constituye la naturaleza dinámica del desarrollo cognitivo, en donde el término dinámica se refiere a los cambios en los mecanismos de desarrollo a lo largo del tiempo.

Según la propuesta de Sternberg, de la misma manera como el desarrollo es un proceso dinámico, la concepción de los psicólogos de los mecanismos del desarrollo que están a la base del cambio intelectual debería ser también dinámica. Los mecanismos de desarrollo deberían ser diferencialmente importantes en diferentes niveles del ciclo de vida del individuo:

En primer lugar, se debería considerar la naturaleza dinámica del procesamiento de información del niño, dado que la literatura nos indica que los procesos metacognitivos deberán ser más importantes en la nińez tardía que en la niñez temprana, ya que los niños mayores invierten relativamente más tiempo planificando las soluciones a los problemas y menos tiempo resolviéndolos. Por lo tanto, si bien las estrategias de construcción de los niños pequeños son difíciles de estudiar y por lo tanto difíciles de comprender la literatura del desarrollo sugiere que para los niños pequeños los procesos no ejecutivos pueden jugar un rol compara- 
tivamente mayor a nivel de adquisición y reorganización del conocimiento, mientras que para los niños mayores, la mejora en el procesamiento ejecutivo puede contribuir al avance en la adquisición y reorganización del conocimiento. Es necesario seguir investigando para esclarecer el rol de los procesos metacognitivos en la niñez temprana.

En segundo lugar, los teóricos del desarrollo dinámicos sugieren que las experiencias tempranas pueden jugar un rol significativo en el desarrollo de experiencias tardías, enfatizando la naturaleza dinámica del cambio sobre el curso de la niñez a la adolescencia. Por otro lado, las ideas sobre el desarrollo en otras áreas pueden ayudar a comprender mejor el desarrollo cognitivo (Flavell, 1984) y es posible que las experiencias de los niños sean diferencialmente importantes para el desarrollo intelectual en diferentes niveles en el tiempo. De la misma manera, la repetición de experiencias y la práctica con determinados problemas son más importantes durante la automatización. Por otro lado, es probable que la experiencia juegue un rol importante en el desarrollo cognitivo antes que el conocimiento comprensivo de un área particular sea adquirido, es decir, las experiencias del nino pueden ejercer una gran influencia en la manera como éste reorganiza su conocimiento cuando conoce relativamente poco sobre un área determinada, más que cuando presenta un conocimiento base ya consolidado en el tema.

Finalmente, el ritmo y la extensión de la ramificación del conocimiento, el último producto del desarrollo cognitivo puede variar en diferentes niveles del ciclo de vida del individuo. El desarrollo durante la adultez difiere del desarrollo durante la infancia y una de las mayores diferencias cognitivas entre los adultos y los niños es el que los adultos presentan mayor nivel de conocimiento que los nifios (Carey, 1985a).

El ritmo y la extensión de adquisición del conocimiento y la reorganización del mismo serán más profundos durante la niñez y adolescencia, ya que es durante estos años que las características estructurales del conocimiento de los adultos son organizadas y consolidadas. 
Por otro lado, determinados eventos en la vida de los niños y adolescentes pueden llevarlos a incorporar grandes cantidades de información, tal como por ejemplo la adquisición del lenguaje, la escolaridad, los estudios superiores, lo cual puede maximizar las ramificaciones de las estructuras del conocimiento durante estos años.

\section{Referencias}

Brainerd, C.J. (1981). Working memory and the developmental analysis of probability judgement, Psychological Review, 88, 463-502.

Carey, S. (1985a). Are children fundamentally different kinds of thinkers and learners than adults? en S.F. Chipman, J.W. Segal \& R. Glaser (eds.), Thinking and Learning Skills: Research and Open Questions, vol.2. Hillsdale, NJ: Erlbaum.

Carey, S. (1985b). Conceptual Change in childhood. Cambridge, MA: MIT Press.

Case, R. (1984). The process of stage transition: A neo-Piagetian view, en R.J. Sternberg (ed.), Mechanisms of Cognitive Development. Nueva York: W.H. Freeman.

Case, R. (1985). Intellectual development: Birth to adulthood. Orlando: Academic Press.

Davidson, J.E. \& Sternberg, R. (1984). The role of insight in intellectual giftedness, Gifted Child Quarterly, 28, 58-64.

Fischer, K. (1980). A theory of cognitive development: The control and construction of hierarchies of skills, Psychological Review, 87, 477531.

Fischer, K.W. \& Pipp, S.L. (1984). Processes of cognitive development: Optimal level and skill acquisition, en R.J. Sternberg (ed.), Mechanisms of Cognitive Development. Nueva York: W.H. Freeman.

Flavell, J.H. (1963). The Developmental Psychology of Jean Piaget. Nueva York: D. Van Nostrand.

Flavell, J.H. (1984). Discussion, en R.J. Sternberg (ed.), Mechanisms of Cognitive Development. Nueva York: W.H. Freeman. 
Ginsburg, H. \& Opper, S. (1979). Piaget's Theory of Intellectual Developmetn: An Introduction (2nd edn.). Englewood Cliffs, NJ: Prentice-Hall. Garrett, H.E. (1946). A developmental theory of intelligence, American Psychologist, 1, 372-378.

Keil, F. (1984). Mechanisms of cognitive development and the structure of knowledge, en R.J. Sternberg (ed.), Mechanisms of Cognitive Development, Nueva York: W.H. Freeman.

Klahr, D. (1984). Introduction: The end of the age of development, en R.J. Sternberg (ed.), Mechanisms of Cognitive Development, Nueva York: W.H. Freeman.

Kohlberg, L. (1969). Stage and sequence: The cognitive-developmental approach to socialization, en D. Goslin (de.), Handbook of Socialization Theory and Research, Chicago: Rand McNally.

Pascual-Leone, J. (1970). A mathematical model for the transition rule in Piaget's developmental stages, Acta Psychologica, 63, 301-345.

Piaget, J. (1976). The psychology of intelligence. Totowa, NJ: Littlefield, Adams.

Rogoff, B. y Wertsch, J. V. (eds) (1984). Children's learning in the 'Zone of Proximal Development', New Directions for Child Development (No.23). San Francisco: Jossey-Bass.

Siegler, R.S. (1984). Mechanisms of cognitive growth: Variation and selection, en R.J. Sternberg (ed.) Mechanisms of Cognitive Development. Nueva York: W.H. Freeman.

Siegler, R.S. (1986a). Children's Thinking. Englewood Cliffs, NJ:Prentice Hall.

Siegler, R.S. (1986b). A panoramic view of development, Contemporary Psychology, 31, 329-331.

Sincoff, J.B. \& Sternberg, R. (1989). The development of cognitive skills. En Colley, A.M. \& Beech, J.R (eds.) Acquisition and performance of cognitive skills, Nueva York: John Wiley \& Sons.

Sternberg, R. (1984). Mechanisms of cognitive development: A componential approach, en R.J. Sternberg (ed.), Mechanisms of Cognitive Development, Nueva York: W.H. Freeman. 
Sternberg, R. (1985). Beyond IQ: A triarchic theory of human intelligence. Cambridge: Cambridge University Press.

Sternberg, R. J. \& Powell, J.S. (1983). The development of intelligence, en P.H. Mussen (series ed. ) y J.H. Flavell y E. Markman (volume eds.), Handbook of Child Psychology (3rd edn), vol. 3. Nueva York: Wiley.

Vygotsky, L.S. (1978) Mind in Society: The development of higher psychological processes. Cambridge: Harvard University Press.

Werner, H. (1948). Comparative psychology of mental development. Nueva York: International Universities Press.

Ziegler, E. \& Glick, M. (1986). A developmental approach to adult psychopathology. Nueva York: Wiley. 